\title{
Morpho-Molecular Study on some Taxa of Apocynaceae Sensu Lato
}

\author{
Mohamed A. Salim ${ }^{1}$, Alsafa H. Mohamed ${ }^{1}$, Mohamed E. Tantawy ${ }^{1}$, Hanan A. Dabbub ${ }^{2}$ and Usama K. Abdel- \\ Hameed ${ }^{1,3 *}$ \\ ${ }^{1}$ Botany Department, Faculty of Science, Ain Shams University, Cairo, Egypt \\ ${ }^{2}$ Biology Department, Faculty of Education, Alzawia University, Zawiya, Libya \\ ${ }^{3}$ Biology Department, College of Science, Taibah University, Al-Madinah, Kingdom of Saudi Arabia \\ *For correspondence: uabdelhameed@taibahu.edu.sa; usama_abdelhameed@sci.asu.edu.eg \\ Received 12 January 2021; Accepted 14 February 2021; Published 16 April 2021
}

\begin{abstract}
Apocynaceae sensu lato (s.l.) is treated as distinct family in some taxonomic systems while in others is treated as two separated families viz. Apocynaceae sensu stricto (s.s.) and Asclepiadaceae. So the main objective of the present study was to adopt one of the two views. The morphological characters viz. whole plant, lamina vein architecture and lamina epidermal characters of 20 wild and ornamental species were examined using LM and SEM. The data were numerically analyzed to detect the phenetic relationship among the studied species. DNA barcoding based on the sequencing of $r b c L$ gene was cladistically analyzed to detect the phylogenetic relationship among the studied species. The generated phenogram showed a clear separation of two subseries; one of them belonged to Apocynaceae and the other with the remaining taxa belonging to Asclepiadaceae. The obtained cladogram showed that all of the studied species were divided into four lineages. It is concluded that the phenetic analysis supports the treatment of Apocynaceae s.l. as two distinct families; Apocynaceae s.s. Asclepiadaceae contrary of the phylogenetic one that supports the treatment of Apocynaceae s.s. and Asclepiadaceae as one family (Apocynaceae s.l.) with four subfamilies viz. Apocynoideae, Rauvolfiodeae, Asclepiadoideae and Periplocoideae. (C) 2021 Friends Science Publishers
\end{abstract}

Keywords: Apocynoideae; Asclepiadoideae; Cladistics; Phenetics; Periplocoideae; Rauvolfiodeae

\section{Introduction}

Apocynaceae and Asclepiadaceae constitute the most diverse lineage of Gentianales and regarded as two closely related families (Civeyrel et al. 1998). Endress et al. (1996) and Sennblad and Bremer (2002) reported that Apocynaceae sensu lato (s.l.) includes the former Asclepiadaceae, and this merge is based on both morphological and molecular similarities. Melchior (1964), Dahlgren (1983) and Jeffrey and Cronquist (1984) separated Asclepiadaceae from Apocynaceae and divided Apocynaceae s.l. into two distinct families recognizing two subfamilies per each; Plumieroideae and Echitoideae within Apocynaceae, while Cynanchoideae and Periplocoideae within Asclepiadaceae.

It has long been known that some taxa of Apocynaceae s.l. are more morphlogically similar to Asclepiadaceae than they are to their own family, a series of morphology-based papers focused on pollen have proved to be the most important morphological character (Endress et al. 2014; Dabbub et al. 2020). The importance of stomatography and leaf venation as a helpful taxonomic tool needs no emphasis, as these characters have immense value in identifying species of Apocynaceae s.l. (Chandra et al
1968). Foliar epidermal characters are considered as very important diagnostic tool to differentiate among the species of the family (Ayaz et al. 2020). Light microscopy (LM) and Scanning electron microscopy (SEM) on leaf of Apocynaceae were believed to have a pivotal role in the classification and delimitation of its species (Bashir et al. 2020).

A wide range of molecular approaches has been used by numerous research groups to find the natural relationship within various groups of Apocynaceae (Potgieter and Albert 2001; Rapini et al. 2003; Rapini et al. 2007; Simões et al. 2007; Livshultz 2010). Fishbein (2001) used chloroplast matk gene and confirmed the monophyly of subfamilies and assumed a closer relationship of Periplocoideae to Apocynaceae s.s. than to the remainder of Asclepiadaceae. Sennblad and Bremer (1996) used the sequence data for rbcL gene of 24 species of Apocynaceae and Asclepiadaceae to evaluate the existing familial and subfamial classification, and concluded that Asclepiadaceae is nested into Apocynaceae. Judd et al. (1994) and Kunze (1996) reported that $r b c L$ gene region did not support the monophyly of Asclepiadaceae and Periplocaceae as separate families, rather indicated a close relationship among Periplocaceae and Apocynaceae s.s. 
A controversy over the taxonomic relationship between the Apocynaceae and Asclepiadaceae persisted. Apocynaceae s.l. has been divided into five subfamilies viz. Apocynoideae, Rauvolfioideae, Asclepiadoideae, Secamonoideae and Periplocoideae. The former two subfamilies belong to Apocynaceae s.s., while the latter three belonging to Asclepiadaceae (Endress et al. 2000; Endress et al. 2007; Angiosperm Phylogeny Group 2016). The principle target of the current investigation was to embrace whether Apocynaceae should be treated as distinct entity or two separated taxonomic entities through the use of some diagnostic morphological characters in phenetic analysis, as well as DNA barcoding based on the sequencing of $r b c L$ gene that is used in phylogenetic analysis.

\section{Materials and Methods}

Twenty species representing four sub-families and 19 genera of Apocynaceae s.l. were collected from natural habitats (wild species) and different botanical gardens (cultivated species) in Egypt and Libya (Table 1). The identification and Synonyms were authenticated based on previous infornation (Bailey 1949; Tackholm 1974; Boulos 2000; IPNI 2012).

\section{Macromorphological characters investigation}

The macromorphological characters of the whole plant viz. habit, stem, leaf and flower were examined, described from the fresh specimens and with the aid of text books (Bailey 1949; Hutchinson and Dalziel 1954; Boulos 2000; Endress and Bruyns 2000; Pandey and Pandey 2006). Huernia andreaeana is with rudimentary leaves, so the lamina vein architecture is not dealt with. Lamina vein architecture is examined from fresh leaves in Nerium oleander, Cynanchum acutum and Carissa carandas, while in other species, leaves were cleaned and soaked in a strong household bleach solution (sodium hypochloride less than $5 \%$, sodium hydroxide less than $5 \%$ and water) until they turn white. The leaves were removed from bleach and rinsed with water then stained with $1 \%$ safranine (Johansen 1940).

Lamina epidermal strips were prepared from fresh leaves of the studied species (leaves rudimentary in $H$. andreaeana). The epidermal strips were prepared by mechanically stripping (Johansen 1940) or by chemical methods (Pohl 1967) by taking fragment of $5-10 \mathrm{~mm}^{2}$ from median portion of leaf, soaked in nitric acid and hydrogen peroxide solution (1:1) for a period ( $2 \mathrm{~h}$ for two days) depending on the leaf thickness. The epidermal strips were stained with $1 \%$ safranine, mounted on slides. Examination and photomicrographs were taken using Canon power-shot A720, 8.0 mega pixels. For SEM investigation, small pieces $\left(7 \mathrm{~mm}^{2}\right)$ of the fresh leaf material were fixed on SEM stubs with double-sided tape, coated with gold in SPI-Module sputter coater. Only abaxial surface was observed and photographed by Scanning Electron Microscope (JSM-5500 LV; JEOL Ltd-Japan) by using high vacuum mode. Terminology of epidermal characteristics was performed with the help of previous information (Metcalfe and Chalk 1950; Ash et al. 1999; Prabhakar 2004; Stearn 2005).

\section{DNA extraction and PCR amplification with rbcL primers}

The fresh leaves of the studied species were collected and subjected to molecular analysis, Solenostemma argel material was practically unavailable and loaded from gene bank (ID HG530567). Leaves (100 mg) of the available material were ground to a powder using liquid nitrogen in Eppendorf tube then DNA were isolated using CTAB (Cetyl-trimethyl ammonium bromide) protocol of (Doyle and Doyle 1987). The purified DNA was used in PCR amplification of $r b c L$ region using universal primers as the following:

\section{Forward primer: 5'-ATG TCA ACA CAA ACA GAG ACT AAA GC-3' \\ Reverse primer: 5'-GAA ACG GTC TAT CCA ACG CAT-3'}

The amplification reactions were performed in $25 \mu \mathrm{L}$ as follow; 5x GoTaq ${ }^{\circledR}$ Flexi buffer $5 \mu \mathrm{L}, \mathrm{MgCl}_{2}(25 \mathrm{mM})$ $2.5 \mu \mathrm{L}$, dNTPs (10 mM each) $0.5 \mu \mathrm{L}$, forward primer (10 $\mu \mathrm{M}) 1.2 \mu \mathrm{L}$, reverse primer $(10 \mu \mathrm{M}) 1.2 \mu \mathrm{L}$, Go Taq ${ }^{\mathrm{TM}}(5$ $\mathrm{U} / \mu \mathrm{L}) 5 \mu \mathrm{L}$, DNA Stock $2 \mu \mathrm{L}, \mathrm{H} 2 \mathrm{O} 7.6 \mu \mathrm{L}$ up to make 25 $\mu \mathrm{L}$ total volume. The reaction conditions were as the following:

Initial denaturation at $95^{\circ} \mathrm{C}$ for 5 min., 40 cycles at $94^{\circ} \mathrm{C}$ for $30 \mathrm{~s}$, $58^{\circ} \mathrm{C}$ for $30 \mathrm{~s}$., $72^{\circ} \mathrm{C}$ for $45 \mathrm{~s}$. and $72^{\circ} \mathrm{C}$ for $10 \mathrm{~min}$. All positive PCR amplicons were prepared for the cleanup step (purification) from other undesired substances as dimers, RNA, free nucleotides and unamplified DNA fragments by using PCR product purification Kit (Thermo PCR purification kit, USA). It is an essential step before automated DNA-sequencing. The purified DNA was submitted for sequencing to Macrogen, Korea; 6F, 172, Dolma-ro, Bundang-gu, Seongnam-si, Gyeonggi-do (Jeongja-dong, Seoul National University Bundang Hospital Healthcare Innovation Park).

\section{Phenetic analysis}

The morphological data of 20 species were subjected to phenetic analysis based on 176 morphological characters states. Prior to analysis the presence for character states were indicated as numerical values in order to make comparison during similarity estimation feasible. Thus, a uniform convention was used for all characters; " 1 " used for present and " 0 " for absent. All computations were carried out using NTSYS-PC version 2.02 software program (Rohlf 2000). 


\section{Phylogenetic analysis}

Crucianella angustifolia (family Rubiaceae) was designed as an out group for phylogenetic analysis and loaded also from gene bank (ID X81094). The sequences were prepared for alignment to get the best trees. The chromatograms in sequences were compiled using BIOEDIT V3 program (Hall 1999). Sequence service chromatogram (fasta file) of sample was imported into a new alignment file created in BIOEDIT. The MEGA7 a phylogenetic program was used for assembling data and constructing the tree. Alignment fasta file was entered into the program for constructing the phylogenetic tree. Tree was constructed according to Neighbor Joining model, which is based on the number of distances between sequences. The number of bootstrap tests was performed using 1000 replicates.

\section{Results}

\section{Phenetic analysis}

A summary of morphological characters states and its codes as revealed by LM \& SEM is presented in Table 2 and some of the most specific structures were illustrated in Fig. 1.

The obtained phenogram (Fig. 2) resulting from the cluster analysis of 176 morphological characters states of whole plant, lamina architecture, stomatography (LM and SEM) showed that $H$. andreaeana basally segregated with unresolved relationship due to the lack of many character states (102 leaf character states). The remaining 19 species were separated into two series; I and II. Series I comprised Acokanthera oblongifolia, Alstonia scholaris, Adenium obesum, C. carandas, C. macrocarpa, Cerbera odollam, Tabernaemontana divaricate, Wrightia coccinea, Kopsia arborea, Plumeria obtusa, Cascabela thevetia, N. oleander, Asclepias curassavica, Cryptostegia grandiflora, Calotropis procera, S. argel, Gomphocarpus sinaicus and C. acutum, at a taxonomic distance 0.38 based on sharing of simple leaf, paracytic or anomocytic stomata, smooth surface anticlinal wall, pinnate primary vein category and regular polygonal reticulate quaternary vein category. Series II included Catharanthus roseus as a single entity at a taxonomic distance 0.38. Owing to annual herb, amphistomatic leaf type, eucamptodromus secondary veins, opposite percurrent tertiary veins with straight course, wrinkled anticlinal wall of epidermal cells.

Series I was divided into two sub-series; (A and B) at a taxonomic distance 0.45 . Sub-series A was divided into two clusters; $\mathrm{C} 1$ and $\mathrm{C} 2$ at a taxonomic distance 0.47 . Cluster 1 was divided into two groups; G1 and G2 at a taxonomic distance 0.51. Group 1 included A. oblongifolia and $A$. scholaris as one group due to presence fissured layers wax with ill-defned sculpture of lamina abaxial epidermal cells and elliptic leaf. Group 2 included nine studied species sharing the presence of narrow, depressed anticlinal walls with smooth surface, and raised periclinal walls.
Cluster 2 included $N$. oleander at a taxonomic distance 0.47 owing to cladodromous secondary veins categoty, sunken stomata and curved anticlinal wall adaxially, incomplete looped marginal veins, one-branched freely ending ultimate veins, granulate crystalloid epicuticular wax and colliculate sculpture of lamina abaxially.

Sub-series B was divided into two clusters; C3 and C4 at a taxonomic distance 0.55 . C 3 was divided into two groups; G3 and G4 at a taxonomic distance 0.60 . Group 3 included A. curassavica and C. grandiflora grouped together as one group. The latter group 4 included $C$. procera, G. sinaicus and $S$. argel based on sharing morphological characters viz. ad medially ramified tertiary veins, amphistomatic leaf type and sunken or leveled stomata elevation abaxially. The latter cluster 4 included $C$. acutum due to presence climbing stem, cordate leaves with actinodromus primary vein category, ill-defned sculpture of lamina abaxially with hight density of non-entire platlets epicuticular.

\section{Phylogenetic analysis}

The obtained sequences were uploaded to Genbank and gained accession numbers as follow; BankIt2396104 A. oblongifolia MW208824, BankIt2396104 A. obesum MW208825, BankIt2396104 A. scholaris MW208826, BankIt2396104 A. curassavica MW208827, BankIt2396104 C. procera MW208828, BankIt2396104 C. carandas MW208829, BankIt2396104 C. macrocarpa MW208830, BankIt2396104 C. thevetia MW208831, BankIt2396104 C. roseus MW208832, BankIt2396104 C. odollam MW208833, BankIt2396104 C. grandiflora MW208834, BankIt2396104 C. acutum MW208835, BankIt2396104 G. sinaicus MW208836, BankIt2396104 H. andreaeana MW208837, BankIt2396104 K. arborea MW208838, BankIt2396104 N. oleander MW208839, BankIt2396104 P. obtusa MW208840, BankIt2396104 T. divaricata MW208841, BankIt2396104 W. coccinea MW208842.

The generated cladogram (Fig. 3), which was rooted by $C$. angustifolia as an outgroup, supported the monophylly of the taxa under investigation, which can be differentiated into four lineages. The first one included $P$. obtusa and $K$. arborea as sister taxa due to sharing of intermarginal secondary veins, well developed areolation, two or more branched freely ending ultimate veins, straight anticlinal wall of $\mathrm{ab} /$ adaxial epidermal cells and leveled stomata (synapomorphic characters). The second lineage included A. oblongifolia, C. carandas, C. macrocarpa, A. scholaris, $C$. odollam and $C$. thevetia. All taxa are nested together at 0.771 bootstrap value after separation of $C$. roseus and $T$. divaricata at earlier basal level. $A$. oblongifolia, C. carandas and C. macrocarpa are nested together at 0.787 bootstrap value based on synapomorphic characters $v i z$. shrub habitat, salver corolla tube and union of carpels (ovary, style and stigma) throughout. In the third lineage, $C$. grandiflora and $W$. coccinea were nested 
Table 1: Data of collection of the studied species

\begin{tabular}{|c|c|c|}
\hline No. & Taxa & Locality/source \\
\hline 1. & $\begin{array}{l}\text { Acokanthera oblongifolia Benth. \& Hook.f. - Gen. Pl. [Bentham \& Hooker f.] 2(2): 696. } 1876 \text { [May 1876]; nom. inval. (IK) = A. } \\
\text { spectabilis (Sond.) Hook.f. }\end{array}$ & $\mathrm{BG}$ \\
\hline 2. & Adenium obesum Roem. \& Schult. - Syst. Veg., ed. 15 bis [Roemer \& Schultes] 4: 411.1819 (IK) = A. arabicum Balf.f & OBG \\
\hline 3. & Alstonia scholaris (L.) R.Br. - Mem. Wern. Nat. Hist. Soc. 1: 75. 1811 [dt. 1809; issued in 1811] (IK) = Echites scholaris L. & GZ \\
\hline 4. & Asclepias curassavica L. - Sp. Pl. 1: 215. 1753 [1 May 1753] (IK) = A. margaritacea Hoffmanns. ex Schult. & OBG \\
\hline 5. & Calotropis procera W.T.Aiton - Hort. Kew., ed. 2 [W.T. Aiton] 2: 78. 1811 (IK) = C. persica Gand. & WML \\
\hline 6. & Carissa carandas L. -- Mant. Pl. 52. 1767 [15-31 Oct 1767] (IK) = C. carandas var. congesta (Wight) Bedd. & BG \\
\hline 7. & Carissa macrocarpa (Eckl.) A. DC. - Prodr. [A. P. de Candolle] 8: 336. 1844 [mid Mar 1844] (IK) = Arduina grandiflora E.Mey. & OBG \\
\hline 8. & Cascabela thevetia (L.) Lippold. - Feddes Repert. 91: 52.1980 (GCI) = Thevetia peruviana (Pers.) K.Schum. & BG \\
\hline 9. & Catharanthus roseus (L.) G.Don. - Gen. Hist. 4(1): 95.1837 (IK) = Vinca rosea L. & AUG \\
\hline 10 & Cerbera odollam Gaertn. - Fruct. Sem. Pl. 2: 193. 1791 (IK) = Odollamia malabarica Raf. & AG \\
\hline 11. & Cryptostegia grandiflora R.Br. - Bot. Reg. 5: t. 435. 1820 [1819 publ. 1820] (IK) = C. grandiflora Roxb. ex R.B. & AMG \\
\hline 12. & Cynanchum acutum L. - Sp. Pl. 1: 212. 1753 [1 May 1753] (IK) = C. excelsum Desf. & $\mathrm{AU}$ \\
\hline 13. & $\begin{array}{l}\text { Gomphocarpus sinaicus Boiss. - Diagn. Pl. Orient. ser. 1, 11: 80. } 1849 \text { [Mar-Apr 1849] (IK) = Asclepias sinaica } \text { (Boiss.) Muschl = } \\
\text { Gomphocarpus schimperi C.Presl }\end{array}$ & SK \\
\hline 14. & Huernia andreaeana (Rauh) L.C.Leach - J. S. African Bot. 40(1): 21. 1974 (IK) = H. appendiculata Berger. & WML \\
\hline 15. & $\begin{array}{l}\text { Kopsia arborea Blume. - Cat. Gew. Buitenzorg (Blume) 13. 1823; et Bijdr. Fl. Ned. Ind. 16: } 1030 \text { [Oct 1826-Nov 1827]. (IK) = K. } \\
\text { jasminiflora Pit. }\end{array}$ & BG \\
\hline 16. & Nerium oleander L. - Sp. Pl. 1: 209. 1753 [1 May 1753] (IK) = N. carneum Dum.Cours. & AUG \\
\hline 17. & Plumeria obtusa L. - Sp. Pl. 1: 210. 1753(IK) = P. apiculata Urb. & AUG \\
\hline 18. & Solenostemma argel Hayne - Getreue Darstell. Gew. ix. t.38. 1825. (IK) = Cynanchum arghel Delile. & A \\
\hline 19. & $\begin{array}{l}\text { Tabernaemontana divaricata (L.) R.Br. ex Roem. \& Schult. - Syst. Veg., ed. } 15 \text { bis [Roemer \& Schultes] 4: } 427.1819 \text { (IK) = } \\
\text { Taberna discolor (Sw.) Miers }\end{array}$ & BG \\
\hline 20. & Wrightia coccinea Sims - Bot. Mag. 53: t. 2696. $1826(\mathrm{IK})=$ Nerium coccineum Roxb. ex Hornem. $=$ N. coccineum Lodd. & AG \\
\hline
\end{tabular}

together at a high bootstrap value (0.792) due to the presence of opposite leaves, weak brochidodromous secondary veins and paracytic lens-shaped stomata (synapomorphic characters). In the last lineage, eight studied species were nested at 0.772 bootstrap value. A. obesum and $N$. oleander were separated consequently at basal level, while A. curassavica, G. sinaicus, C. procera, $C$. acutum, $S$. argel and $H$. andreaeana were nested together at 0.993 bootstrap value. The latter six species shared many synapomrphic characters such as campanulate corolla tube, presence of gynostegium, two carpels (free below and united above) with true pollinia. These species were separated consequently from $H$. andreaeana at basal level due to autapomorphic characters viz. succulent stem, redumintary leaves features in addition to winged pollinium. C. acutum was separated based on autapomorphic characters viz. climbing stem, cordate leaf and erect orientation of pollinium. C. acutum was delimited from all the studied species of Asclepiadeae on the basis of rhizome stem and presence of opposite petiolated leaves, reaching $G$. sinaicus and $A$. curassavica that forming sister species based on synapomorphic characters viz. globose head stigma, oblong-ovate shape of pollinium sac and yellow colour pollinium. $S$. argel is considered a sister group to $C$. procera, A. curassavica and G. sinaicus.

\section{Discussion}

The generated phenogram supports the treatment of Apocynaceae s.l. as two distinct families, Apocynaceae s.s. and Asclepiadaceae where there is a clear separation of series I into two subseries; one of them belongs to Apocynaceae and the other with the remaining taxa belongs to Asclepiadaceae (Brown 1810; Bessey 1915; Hutchinson 1959; Takhtajan 1980; Goldberg 1986; Dabbub et al. 2020). Within Apocynaceae A. oblongifolia and A. scholaris were nested in one group and this is in accordance with (Byng et al. 2016) and (Endress and Bruyns 2000) where they included them within subfamily Rauvolfioideae. Group 2 contained two species from Apocynoideae (A. obesum and $W$. coccinea) and seven species from Rauvolfioideae according to (Byng et al. 2016) and (Endress and Bruyns 2000). Sub-series B was divided into two clusters; C3 and C4. C 3 was divided into two groups; G3 and G4. Group 3 included A. curassavica and C. grandiflora grouped kept together as one group. The latter group 4 included $C$. procera, G. sinaicus and $S$. argel. This group is comparable with tribe Asclepiadeae according to (Melchior 1964; Endress et al. 2014; Byng et al. 2016; El-gazzar et al. 2018).

Phylogentically, P. obtusa and K. arborea showed a sister relationship both belonging to Rauvolfioideae, this is comparable with Melchior (1964) and Sennblad and Bremer (1996) who gathered them under subfamily Plumeroideae (Apocynacese s.s.), while Endress and Bruyns (2000) and Byng et al. (2016) put them in subfamily Rauvolfioideae (Apocynacese s.l.). A. oblongifolia, C. carandas, $C$. macrocarpa, A. scholaris, $C$. odollam and $C$. thevetia were nested together (Rauvolfioideae). While $C$. roseus and $T$. divaricata were separated at earlier basal level; the pattern of separation clarified that Rauvolfioideae is not monophyletic group (paraphyletic). (Simões et al. 2007) reported six monophyletic tribes within Rauvolfioideae viz. 
Morpho-Molecular Study on some Taxa of Apocynaceae / Intl J Agric Biol, Vol 25, No 5, 2021

Table 2: Morphological character, states (176) and its codes of the studied species used for phenetic analysis. $(0=$ Absent; $1=$ Present $)$

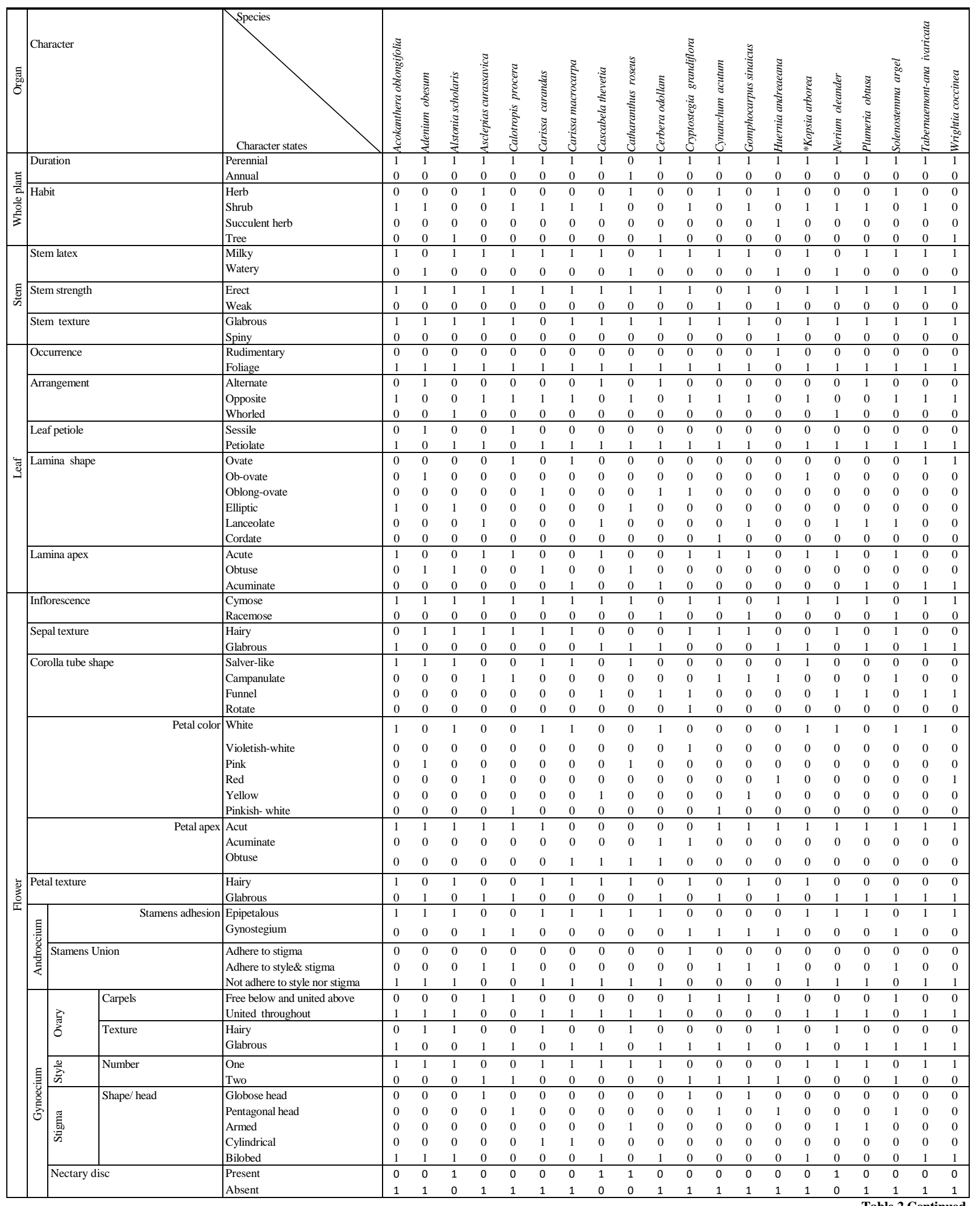


Salim et al. / Intl J Agric Biol, Vol 25, No 5, 2021

Table 2: Continued

\begin{tabular}{|c|c|c|c|c|c|c|c|c|c|c|c|c|c|c|c|c|}
\hline & $1^{\circ}$ vein category & Pinnate & 1 & 1 & 1 & 1 & 1 & 1 & 1 & 1 & 1 & 1 & 1 & 0 & 1 & 0 \\
\hline & & Basal actinodromous & 0 & 0 & 0 & 0 & 0 & 0 & 0 & 0 & 0 & 0 & 0 & 1 & 0 & 0 \\
\hline & $2^{\circ}$ vein category & Weak brochidodromou & 0 & 0 & 0 & 0 & 0 & 0 & 0 & 0 & 0 & 0 & 1 & 0 & 1 & 0 \\
\hline & & Festooned brochidodromous & 1 & 0 & 0 & 0 & 0 & 1 & 0 & 0 & 0 & 0 & 0 & 1 & 0 & 0 \\
\hline & & Brochidodromous & 0 & 1 & 0 & 1 & 1 & 0 & 1 & 0 & 0 & 0 & 0 & 0 & 0 & 0 \\
\hline & & Eucamptodromous & 0 & 0 & 0 & 0 & 0 & 0 & 0 & 0 & 1 & 0 & 0 & 0 & 0 & 0 \\
\hline & & Intramarginal vein & 0 & 0 & 1 & 0 & 0 & 0 & 0 & 1 & 0 & 1 & 0 & 0 & 0 & 0 \\
\hline & & Cladodromous & 0 & 0 & 0 & 0 & 0 & 0 & 0 & 0 & 0 & 0 & 0 & 0 & 0 & 0 \\
\hline & $2^{\circ}$ vein spacing & Decreasing toward base & 1 & 0 & 0 & 0 & 0 & 0 & 0 & 0 & 0 & 0 & 1 & 0 & 0 & 0 \\
\hline & & Increasing toward base & 0 & 0 & 0 & 0 & 0 & 0 & 0 & 0 & 0 & 0 & 0 & 0 & 0 & 0 \\
\hline & & Irregular & 0 & 0 & 0 & 0 & 0 & 1 & 1 & 0 & 0 & 0 & 0 & 1 & 0 & 0 \\
\hline & & Uniform & 0 & 1 & 1 & 1 & 1 & 0 & 0 & 1 & 1 & 1 & 0 & 0 & 1 & 0 \\
\hline & $2^{\circ}$ vein angle & Uniform & 0 & 1 & 1 & 0 & 1 & 0 & 0 & 1 & 1 & 0 & 0 & 1 & 0 & 0 \\
\hline & & Smoothly decrease toward base & 1 & 0 & 0 & 1 & 0 & 0 & 0 & 0 & 0 & 0 & 1 & 0 & 0 & 0 \\
\hline & & Smoothly increase toward base & 0 & 0 & 0 & 0 & 0 & 1 & 1 & 0 & 0 & 1 & 0 & 0 & 1 & 0 \\
\hline & Inter- $2^{\circ}$ vein & Absent & 0 & 0 & 1 & 0 & 1 & 0 & 0 & 0 & 0 & 0 & 0 & 0 & 0 & 0 \\
\hline & & Weak & 0 & 0 & 0 & 1 & 0 & 1 & 0 & 1 & 0 & 0 & 1 & 1 & 1 & 0 \\
\hline$\cong$ & & Strong & 1 & 1 & 0 & 0 & 0 & 0 & 1 & 0 & 1 & 1 & 0 & 0 & 0 & 0 \\
\hline 苞 & $3^{\circ}$ vein category & Regular polygonal reticulate & 0 & 1 & 0 & 1 & 0 & 1 & 0 & 0 & 0 & 0 & 1 & 0 & 0 & 0 \\
\hline 를 & & Random reticulate & 1 & 0 & 0 & 0 & 0 & 0 & 1 & 1 & 0 & 1 & 0 & 0 & 0 & 0 \\
\hline$\underset{\text { ప }}{\tilde{z}}$ & & Alternate percurrent & 0 & 0 & 1 & 0 & 1 & 0 & 0 & 0 & 0 & 0 & 0 & 1 & 1 & 0 \\
\hline 1. & & Opposite percurrent & 0 & 0 & 0 & 0 & 0 & 0 & 0 & 0 & 1 & 0 & 0 & 0 & 0 & 0 \\
\hline 氙 & & Mixed opp/alt & 0 & 0 & 0 & 0 & 0 & 0 & 0 & 0 & 0 & 0 & 0 & 0 & 0 & 0 \\
\hline & $3^{\circ}$ vein course & Exmedially & 1 & 0 & 0 & 0 & 0 & 0 & 0 & 0 & 0 & 0 & 1 & 0 & 0 & 0 \\
\hline & & Admedially ramified & 0 & 1 & 1 & 1 & 1 & 1 & 1 & 1 & 0 & 1 & 0 & 1 & 1 & 0 \\
\hline & & Straight & 0 & 0 & 0 & 0 & 0 & 0 & 0 & 0 & 1 & 0 & 0 & 0 & 0 & 0 \\
\hline & & Sinuous & 0 & 0 & 0 & 0 & 0 & 0 & 0 & 0 & 0 & 0 & 0 & 0 & 0 & 0 \\
\hline & $4^{\circ}$ vein category & IIl-developed & 0 & 0 & 0 & 0 & 0 & 0 & 0 & 0 & 1 & 0 & 0 & 0 & 0 & 0 \\
\hline & & Regular polygonal reticulate & 1 & 1 & 1 & 1 & 1 & 1 & 1 & 1 & 0 & 1 & 1 & 1 & 1 & 0 \\
\hline & $5^{\circ}$ vein category & IIl-developed & 1 & 0 & 0 & 0 & 1 & 0 & 0 & 1 & 1 & 0 & 0 & 0 & 1 & 0 \\
\hline & & Regular polygonal & 0 & 0 & 0 & 0 & 0 & 0 & 1 & 0 & 0 & 0 & 1 & 0 & 0 & 0 \\
\hline & & Dichotomizing & 0 & 1 & 1 & 1 & 0 & 1 & 0 & 0 & 0 & 1 & 0 & 1 & 0 & 0 \\
\hline & Aereolation & Lacking & 1 & 1 & 1 & 1 & 1 & 0 & 0 & 0 & 1 & 1 & 1 & 1 & 1 & 0 \\
\hline & & Well-developed & 0 & 0 & 0 & 0 & 0 & 1 & 1 & 0 & 0 & 0 & 0 & 0 & 0 & 0 \\
\hline & & Poorly developed & 0 & 0 & 0 & 0 & 0 & 0 & 0 & 1 & 0 & 0 & 0 & 0 & 0 & 0 \\
\hline & Free ending ultimate vein & Absent & 1 & 0 & 0 & 0 & 1 & 0 & 1 & 1 & 1 & 0 & 0 & 0 & 1 & 0 \\
\hline & & 1- branched & 0 & 0 & 0 & 0 & 0 & 1 & 0 & 0 & 0 & 0 & 1 & 0 & 0 & 0 \\
\hline & & 2- or more branched & 0 & 1 & 1 & 1 & 0 & 0 & 0 & 0 & 0 & 1 & 0 & 1 & 0 & 0 \\
\hline & \begin{tabular}{|l|} 
Marginal ultimate venation \\
\end{tabular} & Looped & 1 & 1 & 0 & 1 & 1 & 1 & 1 & 1 & 1 & 1 & 1 & 1 & 0 & 0 \\
\hline & & Incomplete loop & 0 & 0 & 1 & 0 & 0 & 0 & 0 & 0 & 0 & 0 & 0 & 0 & 1 & 0 \\
\hline & Leaf type & Ill-defined & 0 & 0 & 0 & 0 & 0 & 0 & 0 & 0 & 0 & 0 & 0 & 0 & 0 & 0 \\
\hline & & Hypostomatic & 1 & 1 & 1 & 1 & 0 & 1 & 1 & 1 & 0 & 1 & 1 & 1 & 0 & 0 \\
\hline & & Amphistomatic & 0 & 0 & 0 & 0 & 1 & 0 & 0 & 0 & 1 & 0 & 0 & 0 & 1 & 0 \\
\hline & Abaxial cell shape & Polygonal & 1 & 1 & 1 & 0 & 1 & 0 & 0 & 0 & 0 & 0 & 1 & 0 & 1 & 0 \\
\hline & & Irregular & 0 & 0 & 0 & 1 & 0 & 1 & 1 & 1 & 1 & 1 & 0 & 1 & 0 & 0 \\
\hline & Abaxial anticlinal walls & Straight & 0 & 1 & 1 & 0 & 1 & 0 & 0 & 0 & 0 & 0 & 1 & 0 & 1 & 0 \\
\hline & & Curved & 1 & 0 & 0 & 0 & 0 & 1 & 1 & 0 & 0 & 0 & 0 & 0 & 0 & 0 \\
\hline$\hat{\Sigma}$ & & Undulate & 0 & 0 & 0 & 1 & 0 & 0 & 0 & 1 & 1 & 1 & 0 & 1 & 0 & 0 \\
\hline e & Adaxial cell shape & Polygonal & 1 & 1 & 1 & 1 & 1 & 1 & 1 & 0 & 1 & 0 & 1 & 1 & 1 & 0 \\
\hline$\tilde{E}$ & & Irregular & 0 & 0 & 0 & 0 & 0 & 0 & 0 & 1 & 0 & 1 & 0 & 0 & 0 & 0 \\
\hline 岛 & Adaxial anticlinal walls & Straight & 1 & 1 & 1 & 1 & 1 & 1 & 1 & 0 & 1 & 1 & 1 & 1 & 1 & 0 \\
\hline$\frac{\pi}{0}$ & & Curved & 0 & 0 & 0 & 0 & 0 & 0 & 0 & 0 & 0 & 0 & 0 & 0 & 0 & 0 \\
\hline$\Xi$ & & Undulate & 0 & 0 & 0 & 0 & 0 & 0 & 0 & 1 & 0 & 0 & 0 & 0 & 0 & 0 \\
\hline E & \begin{tabular}{|l|} 
Abaxial stomatal type \\
\end{tabular} & Wanting & 0 & 0 & 1 & 0 & 0 & 0 & 0 & 0 & 0 & 0 & 0 & 0 & 0 & 0 \\
\hline 券 & & Paracytic & 1 & 1 & 0 & 0 & 0 & 1 & 0 & 0 & 0 & 0 & 1 & 0 & 0 & 0 \\
\hline$\underset{\tilde{z}}{\tilde{z}}$ & & Anomocytic & 0 & 0 & 0 & 1 & 1 & 0 & 1 & 0 & 1 & 0 & 0 & 1 & 1 & 0 \\
\hline 嗮 & & Anisocytic & 0 & 0 & 0 & 0 & 0 & 0 & 0 & 0 & 1 & 1 & 0 & 0 & 0 & 0 \\
\hline 3 & & Sunkun & 0 & 0 & 0 & 0 & 0 & 0 & 0 & 0 & 0 & 0 & 0 & 0 & 0 & 0 \\
\hline & & Paracytic\& Anisocytic & 0 & 0 & 0 & 0 & 0 & 0 & 0 & 1 & 0 & 0 & 0 & 0 & 0 & 0 \\
\hline & \begin{tabular}{|l|} 
Adaxial stomatal type \\
\end{tabular} & Wanting & 1 & 1 & 1 & 1 & 0 & 1 & 1 & 1 & 0 & 1 & 1 & 1 & 0 & 0 \\
\hline & & Anomocytic & 0 & 0 & 0 & 0 & 1 & 0 & 0 & 0 & 0 & 0 & 0 & 0 & 1 & 0 \\
\hline & & Anisocytic & 0 & 0 & 0 & 0 & 0 & 0 & 0 & 0 & 1 & 0 & 0 & 0 & 0 & 0 \\
\hline & Trichomes & Wanting & 1 & 0 & 1 & 1 & 1 & 1 & 1 & 1 & 0 & 1 & 1 & 1 & 0 & 0 \\
\hline & & Eglandular & 0 & 1 & 0 & 0 & 0 & 0 & 0 & 0 & 1 & 0 & 0 & 0 & 0 & 0 \\
\hline & & Glandular (clavate) & 0 & 0 & 0 & 0 & 0 & 0 & 0 & 0 & 0 & 0 & 0 & 0 & 1 & 0 \\
\hline & Epicuticular wax & Striated & 0 & 0 & 0 & 0 & 1 & 0 & 0 & 0 & 0 & 0 & 0 & 0 & 0 & 0 \\
\hline & & Fissured layers & 1 & 0 & 1 & 0 & 0 & 0 & 0 & 0 & 0 & 0 & 0 & 0 & 0 & 0 \\
\hline & & Smooth film & 0 & 0 & 0 & 0 & 0 & 1 & 1 & 1 & 0 & 1 & 0 & 0 & 1 & 0 \\
\hline & & Granulate crystalloid & 0 & 0 & 0 & 0 & 0 & 0 & 0 & 0 & 0 & 0 & 0 & 0 & 0 & 0 \\
\hline & & Crystalloid threads & 0 & 1 & 0 & 1 & 0 & 0 & 0 & 0 & 1 & 0 & 1 & 0 & 0 & 0 \\
\hline & & Non entire platelets & 0 & 0 & 0 & 0 & 0 & 0 & 0 & 0 & 0 & 0 & 0 & 1 & 0 & 0 \\
\hline$\sum_{i=1}$ & Sculpture & Rugose & 0 & 0 & 0 & 0 & 0 & 0 & 0 & 0 & 0 & 0 & 0 & 0 & 0 & 0 \\
\hline 实 & & Ruminate & 0 & 1 & 0 & 1 & 1 & 1 & 1 & 1 & 1 & 1 & 1 & 0 & 1 & 0 \\
\hline 起 & & Ill-defined & 1 & 0 & 1 & 0 & 0 & 0 & 0 & 0 & 0 & 0 & 0 & 1 & 1 & 0 \\
\hline 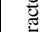 & & Reticulate-foveate & 0 & 0 & 0 & 0 & 0 & 0 & 0 & 0 & 0 & 0 & 0 & 0 & 0 & 0 \\
\hline 吾 & & Colliculate & 0 & 0 & 0 & 0 & 0 & 0 & 0 & 0 & 0 & 0 & 0 & 0 & 0 & 0 \\
\hline$\Xi$ & Abaxial anticlinal walls width & Not obvious & 1 & 0 & 1 & 0 & 0 & 0 & 0 & 0 & 0 & 0 & 0 & 1 & 0 & 0 \\
\hline ह & & Narrow & 0 & 1 & 0 & 1 & 1 & 1 & 1 & 1 & 1 & 1 & 1 & 0 & 1 & 0 \\
\hline 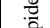 & & Broad & 0 & 0 & 0 & 0 & 0 & 0 & 0 & 0 & 0 & 0 & 0 & 0 & 0 & 0 \\
\hline$\underset{\Xi}{\Xi}$ & Abaxial anticlinal walls & Not obvious & 1 & 0 & 1 & 0 & 0 & 0 & 0 & 0 & 0 & 0 & 0 & 1 & 0 & 0 \\
\hline 春 & elevation & Raised & 0 & 1 & 0 & 0 & 0 & 0 & 0 & 0 & 0 & 0 & 0 & 0 & 0 & 0 \\
\hline है & & Depressed & 0 & 0 & 0 & 1 & 1 & 1 & 1 & 1 & 1 & 1 & 1 & 0 & 1 & 0 \\
\hline
\end{tabular}

Table 2: Continued 
Table 2: Continued

\begin{tabular}{|c|c|c|c|c|c|c|c|c|c|c|c|c|c|c|c|c|c|c|c|c|c|c|}
\hline \multirow{3}{*}{\multicolumn{2}{|c|}{ Abaxial anticlinal wall surface }} & Ill-defined & 1 & 0 & 1 & 0 & 0 & 0 & 0 & 0 & 0 & 0 & 0 & 1 & 0 & 0 & 0 & 0 & 0 & 0 & 0 & 0 \\
\hline & & Smooth & 0 & 1 & 0 & 1 & 1 & 1 & 1 & 1 & 0 & 1 & 1 & 0 & 1 & 0 & 1 & 1 & 1 & 1 & 1 & 1 \\
\hline & & Wrinkled & 0 & 0 & 0 & 0 & 0 & 0 & 0 & 0 & 1 & 0 & 0 & 0 & 0 & 0 & 0 & 0 & 0 & 0 & 0 & 0 \\
\hline \multirow{3}{*}{\multicolumn{2}{|c|}{ Abaxial periclinal wall elevation }} & Ill-defined & 1 & 0 & 1 & 0 & 0 & 0 & 0 & 0 & 0 & 0 & 0 & 1 & 0 & 0 & 0 & 0 & 0 & 0 & 0 & 0 \\
\hline & & Raised & 0 & 0 & 0 & 1 & 1 & 1 & 1 & 1 & 1 & 1 & 1 & 0 & 1 & 0 & 1 & 1 & 1 & 1 & 1 & 1 \\
\hline & & Depressed & 0 & 1 & 0 & 0 & 0 & 0 & 0 & 0 & 0 & 0 & 0 & 0 & 0 & 0 & 0 & 0 & 0 & 0 & 0 & 0 \\
\hline \multirow{4}{*}{\multicolumn{2}{|c|}{ Abaxial periclinal wall surface }} & Ill-defined & 1 & 0 & 1 & 0 & 0 & 0 & 0 & 0 & 0 & 0 & 0 & 1 & 0 & 0 & 0 & 0 & 0 & 0 & 0 & 0 \\
\hline & & Smooth & 0 & 1 & 0 & 1 & 1 & 1 & 1 & 1 & 1 & 1 & 0 & 0 & 1 & 0 & 1 & 0 & 0 & 0 & 1 & 1 \\
\hline & & Wrinkled & 0 & 0 & 0 & 0 & 0 & 0 & 0 & 0 & 0 & 0 & 1 & 0 & 0 & 0 & 0 & 0 & 1 & 1 & 0 & 0 \\
\hline & & Granulated & 0 & 0 & 0 & 0 & 0 & 0 & 0 & 0 & 0 & 0 & 0 & 0 & 0 & 0 & 0 & 1 & 0 & 0 & 0 & 0 \\
\hline \multirow{9}{*}{ 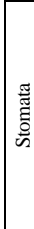 } & \multirow{6}{*}{ Shape } & Circular & 1 & 0 & 0 & 0 & 0 & 0 & 0 & 0 & 0 & 0 & 0 & 0 & 0 & 0 & 1 & 0 & 0 & 0 & 0 & 0 \\
\hline & & Lens-shaped & 0 & 1 & 0 & 0 & 0 & 0 & 0 & 0 & 1 & 0 & 1 & 1 & 1 & 0 & 0 & 0 & 0 & 0 & 0 & 1 \\
\hline & & Elliptic & 0 & 0 & 1 & 1 & 0 & 0 & 0 & 0 & 0 & 0 & 0 & 0 & 0 & 0 & 0 & 0 & 1 & 1 & 0 & 0 \\
\hline & & Ovate & 0 & 0 & 0 & 0 & 1 & 0 & 0 & 1 & 0 & 0 & 0 & 0 & 0 & 0 & 0 & 0 & 0 & 0 & 0 & 0 \\
\hline & & Semi-circular & 0 & 0 & 0 & 0 & 0 & 1 & 1 & 0 & 0 & 1 & 0 & 0 & 0 & 0 & 0 & 0 & 0 & 0 & 1 & 0 \\
\hline & & Sunken in groove & 0 & 0 & 0 & 0 & 0 & 0 & 0 & 0 & 0 & 0 & 0 & 0 & 0 & 0 & 0 & 1 & 0 & 0 & 0 & 0 \\
\hline & \multirow[t]{3}{*}{ Elevation } & Leveled & 1 & 0 & 0 & 0 & 0 & 1 & 0 & 0 & 1 & 1 & 0 & 0 & 0 & 0 & 1 & 0 & 1 & 1 & 1 & 1 \\
\hline & & Sunken & 0 & 0 & 0 & 0 & 1 & 0 & 1 & 0 & 0 & 0 & 0 & 1 & 1 & 0 & 0 & 1 & 0 & 0 & 0 & 0 \\
\hline & & Raised & 0 & 1 & 0 & 1 & 0 & 0 & 0 & 1 & 0 & 0 & 1 & 0 & 0 & 0 & 0 & 0 & 0 & 0 & 0 & 0 \\
\hline
\end{tabular}

Alyxieae, Carisseae, Hunterieae, Plumerieae, Tabernaemontaneae and Willughbeieae. On the other hand, phylogenetic studies reported the non-monophyletic groups in Rauvolfioideae (Potgieter and Albert 2001). The subfamily Plumerioideae of the Apocynaceae has often been interpreted as a paraphyletic group, from which the other subfamilies of the Apocynaceae have evolved (Fallen 1986; Endress et al. 2014). A. oblongifolia, C. carandas and $C$. macrocarpa are nested together (tribe Carissae) at 0.787 bootstrap value, which is inaccordance with the previous reports (Pichon et al. 1950; Endress and Bruyns 2000; Sennblad and Bremer 2002; Endress et al. 2014; Byng et al. 2016). Likwise, C. grandiflora (Priplocoideae) and $W$. coccinea (Apocynoideae) were nested together contrary of Melchior (1964), Endress et al. (2014) and El-gazzar et al. (2018) whom separated them; W. coccinea in Apocynoideae and $C$. grandiflora in Priplocoideae.

A. obesum and $N$. oleander (Apocynoideae) were separated at basal level, while A. curassavica, G. sinaicus, $C$. procera, $C$. acutum, $S$. argel and $H$. andreaeana (Asclepiadoideae) were clustered together. This clade is corresponding to tribe Stapeliae passing with $C$. acutum as a separate clade (Chase et al. 2016), C. acutum is delimited from all the studied species of Asclepiadeae, reaching $G$. sinaicus and $A$. curassavica that forming sister species (Sennblad and Bremer 1996). S. argel (Glossonematinae) is considered a sister group to C. procera, A. curassavica and G. sinaicus (Asclepiadinae) (Melchior 1964). From the pattern of the fourth lineage, there is a strong support to the monophylly of tribe Asclepiadoideae (Chase et al. 2016; Elgazzar et al. 2018).

Melchior (1964) divided Apocynaceae s. $l$. into two distinct families and two sub-families for each; Apocynaceae (Plumieroideae and Echitoideae) and Asclepiadaceae (Cynanchoideae and Periplocoideae). Apocynaceae and Asclepiadaceae have been treated as one family and are recognized five sub-families; three of Asclepiadaceae (Periplocoideae, Asclepiadoideae and Secamonoideae) and two of Apocynaceae s.s. (Rauvolfiodeae and Apocynoideae) (Endress 2004; Endress et al. 2007; Endress et al. 2014;
Chase et al. 2016). The obtained cladogram nested studied species of Asclepiadoideae in a separate lineage and the remaining studied species of Periplocoideae, Apocynoideae and Rauvolfioideae into three lineages. The phylogenetic analysis in the present study supports the treatment of Apocynaceae and Asclepiadaceae as one family (Apocynaceae s.l.) with four subfamilies, which is accirding to previous reports (Endress 2004; Endress et al. 2014; Chase et al. 2016).

\section{Conclusion}

The phenetic analysis in the present study supports the treatment of Apocynaceae s.l. as two distinct families Apocynaceae s.s. and Asclepiadaceae contrary of the phylogenetic one that supports the treatment of Apocynaceae s.s. and Asclepiadaceae as one family (Apocynaceae s.l.) with four subfamilies viz. Apocynoideae, Rauvolfiodeae, Asclepiadoideae and Periplocoideae.

\section{Author Contributions}

MS drafted the manuscript and statistically analyzed the data, AM analyzed and interpreted the results, MT planned the study conception and design and made critical revision, HD acquainted the data and UA made illustrations, made the write up and responded to journal reviewers.

\section{Conflict of Interest}

The authors declare that they have no known competing financial interests or personal relationships that could have appeared to influence the work reported in this paper.

\section{Data Availability Declaration}

Authors declare that all data are available upon request from corresponding author 


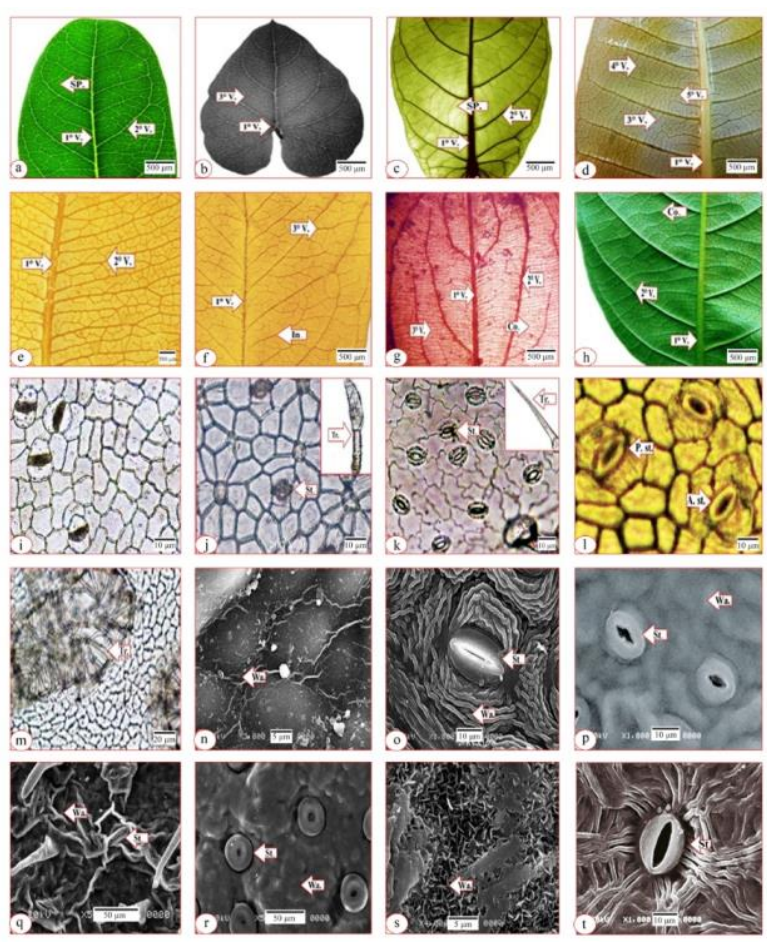

Fig. 1: (a- h): Major categories of lamina vein architecture. a. $C$. carandus, b. C. acutum, c. C. procera, d. P. obtusa, e. K. arborea, f. A. oblongifolia, g. C. roseus, h. C. grandiflora; (i$\mathrm{m})$ : Major aspects of lamina epidermal characteristics (LM). i. A. oblongifolia, j. G, sinaicus, k. C. roseus, 1. P. obtusa, m. N. oleander; ( $\mathrm{n}-\mathrm{t})$ : Major aspects of lamina epidermal characteristics (SEM). n. N. oleander, o. P. obtusa, p. C. carandus, q. A. obesum, r. A. oblongifolia, s. C. acutum, t. C. procera. $1^{\circ}$ V.; Primary vein, $2^{\circ}$ V.; Secondary vein, $3^{\circ}$ V.; Tertiary vein, $4^{\circ} \mathrm{V}$.; Quaternary vein, $5^{\circ} \mathrm{V}$.; Quinary vein, A.St.; Anisocytic stomata, P.St.; Paracytic stomata, Co.; Course; In.; Intersecondaries, Sp.; Space, St.; Stomata. Tr.; Trichome, Wa.; Wax

\section{Conformation to Ethical Guidelines}

All material that were used in the current research do not need to ethically approved, human or animal materials not included

\section{References}

Angiosperm Phylogeny Group (2016). APG IV. Bot J Linn Soc 181:1-20 Ash A, B Ellis, LJ Hickey, K Johnson, P Wilf, S Wing (1999). Manual of Leaf Architecture, Morphological Description and Categorization of Dicotyledonous And Net-Veined Monocotyledonous Angiosperms, Working Group, p:65. Smithsonian Institution, Washington DC, USA

Ayaz A, W Zaman, F Ullah, S Saqib, S Jamshed, S Bahadur, A Shakoor, B Arshad (2020). Systematics study through scanning electron microscopy; a tool for the authentication of herbal drug Mentha suaveolens Ehrh. Microsc Res Tech 83:81-87

Bailey L (1949). Manual of Cultivated Plants. Macmillan Co., New York, USA Bashir K, A Sohail, U Ali, A Ullah, Z Ul Haq, B Gul, I Ullah, Sunera, M Asghar (2020). Foliar micromorphology and its role in identification of the Apocynaceae taxa. Microsc Res Tech 83:755-766

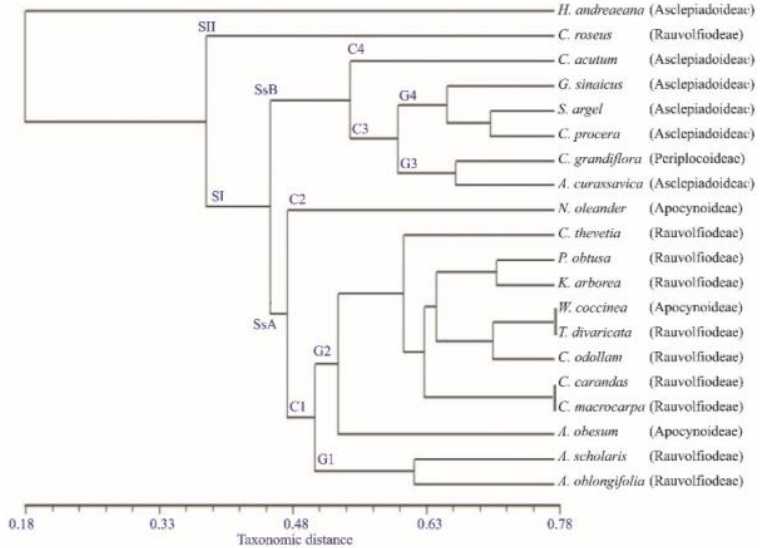

Fig. 2: Phenogram based on 167 morphological characters of the studied taxa of Apocynacaeae s.s. S; series, Ss; subseries, C; cluster, G; group

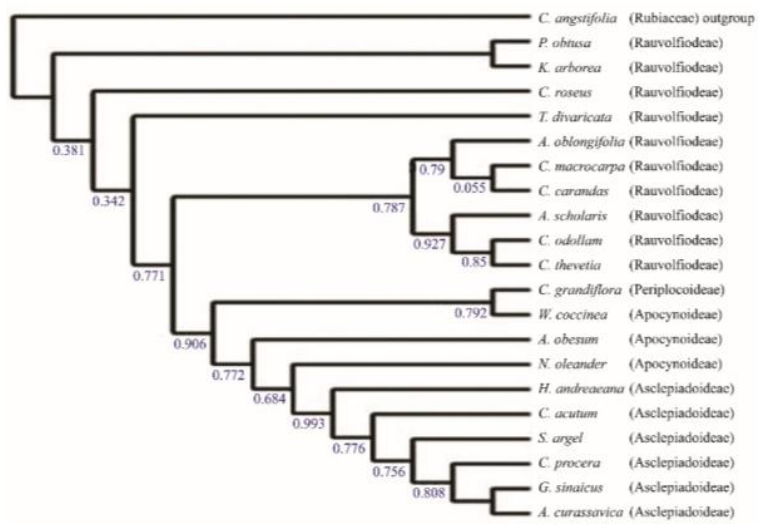

Fig. 3: Neighbor joining phylogenetic tree of the studied taxa of Apocynacaeae s.s. based on chloroplast $r b c \mathrm{~L}$ sequence

Bessey CE (1915). The phylogenetic taxonomy of flowering plants. Ann Missouri Bot Gard 2:109-164

Boulos L (2000). Flora of Egypt VII Al Hadara Publishing Cairo, Egypt

Brown R (1810). On the Asclepiadeae: A Natural Order of Plants Separated from the Apocineae of Jussieu. Cambridge University Press, Cambridge, UK

Byng J, MW Chase, MJM Christenhusz, MF Fay, JW Byng, WS Judd, DE Soltis, DJ Mabberley, AN Sennikov, PS Soltis, PF Stevens (2016). An update of the Angiosperm Phylogeny Group classification for the orders and families of flowering plants: APG IV. Bot J Linn Soc $181: 1-20$

Chandra V, SL Kapoor, C Sharma (1968). Epidermal and venation studies in Apocynaceae - 1. Bul Bot Surv Ind 11:286-289

Chase MW, MJM Christenhusz, MF Fay, JW Byng, WS Judd, DE Soltis, DJ Mabberley, AN Sennikov, PS Soltis, PF Stevens (2016). An update of the Angiosperm Phylogeny Group classification for the orders and families of flowering plants: APG IV. Bot J Linn Soc 181:1-20

Civeyrel L, A Le Thomas, K Ferguson, MW Chase (1998). Critical Reexamination of Palynological Characters Used to Delimit Asclepiadaceae in Comparison to the Molecular Phylogeny Obtained from PlastidmatK Sequences. Mol Phylogenet Evol 9:517-527

Dabbub HA, MA Salim, UK Abdelhameed, ME Tantawy, AH Mohamed (2020). A contribution of the palynological criteria in evaluating the relationships among some species of apocynaceae sensu lato. Egypt J Bot 60:437-449 
Dahlgren R (1983). General aspects of angiosperm evolution and macrosystematics. Nord J Bot 3:119-149

Doyle JJ, JL Doyle (1987). A rapid DNA isolation procedure for small quantities of fresh leaf tissue. Phytochem Bull 19:11-15

El-gazzar A, A Khattab, A El-Saeid, A Elkady (2018). Numerical taxonomy of the Asclepiadaceae s.l. Egypt J Bot 58:321-330

Endress ME (2004). Apocynaceae: Brown and now. Telopea 10:525-541

Endress ME, PV Bruyns (2000). A revised classification of the Apocynaceae s.1. Bot Rev 66:1-56

Endress ME, S Liede-Schumann, U Meve (2007). Advanced in Apocynaceae: The enlightenment, an introduction. Ann Missouri Bot Gard 94:259-267

Endress ME, S Liede-Schumann, U Meve (2014). An updated classification for Apocynaceae. Phytotaxa 159:175-194

Endress ME, B Sennblad, S Nilsson, L Civeyrel, M Chase, S Huysmans, E Grafström, B Bremer (1996). A phylogenetic analysis of Apocynaceae s. str. and some related taxa in Gentianales. A multidisciplinary approach. Opera Bot Belgica 7:59-102

Fallen ME (1986). Floral structure in the Apocynaceae: Morphological, functional, and evolutionary aspects. Bot Jahrbucher Syst Pflanzengeschichte Pflanzengeographie 106:245-286

Fishbein M (2001). Evolutionary innovation and diversification in the flowers of Asclepiadaceae. Ann Missouri Bot Gard 88:603-623

Goldberg A (1986). Classification, evolution, and phylogeny of the families of dicotyledons. Smithson Contribution to Botany pp:1-134

Hall TA (1999). BioEdit: A user-friendly biological sequence alignment editor and analysis program for Windows 95/98/NT. Nucleic Acids Symp Ser 41:95-98

Hutchinson J (1959). The Families of Flowering Plants, Clarendon Press, Oxford, UK

Hutchinson J, JM Dalziel (1954). Flora of West tropical Africa. Crown Agents for the Colonies, London, UK

IPNI (2012). The International Plant Names Index. Available at: https://www.ipni.org/

Jeffrey C, A Cronquist (1984). An integrated system of classification of flowering plants. Kew Bull 38:675

Johansen DA (1940). Plant Microtechnique, McGraw-Hill Book Company, Inc. London

Judd WS, RW Sanders, MJ Donoghue (1994). Angiosperm family pairs: Preliminary phylogenetic analyses. Harvard Pap Bot 1:1-51

Kunze H (1996). Morphology of the stamen in the Asclepiadaceae and its systematic relevance.(With 99 figures in the text). Bot Jahrbucher Syst Pflanzengeschichte Pflanzengeographie 118:547
Livshultz T (2010). The phylogenetic position of milkweeds (Apocynaceae subfamilies Secamonoideae and Asclepiadoideae): Evidence from the nucleus and chloroplast. Taxon 59:1016-1030

Melchior H (1964). Engler's Syllabus der Pflanzenfamilien, Gerbruder Borntraeger, Berlin, West Germany

Metcalfe CR, L Chalk (1950). Anatomy of the Dicotyledons: Leaves Stem and Wood in Relation to Taxonomy, with Notes on Economic Uses: Volume II, Clarendon Press, Oxford, UK

Pandey BP, BP Pandey (2006). A Textbook of Botany Anglosperms. S. Chand Ltd, Jalandhar, India

Pichon M, Others (1950). Classification of the Apocynaceae. XXV. Echitoideae and supplement to the Plumeroideae. Mem Museum Natl d'Histoire Nat Paris Ser B Bot 1:1-170

Pohl RW (1967). Controlled maceration of grass leaves in 40-80\% nitric acid for preparation of epidermis for slides. Stain Technol 42:195-197

Potgieter K, VA Albert (2001). Phylogenetic relationships within Apocynaceae sl based on trnL intron and trnL-F spacer sequences and propagule characters. Ann Missouri Bot Gard 88:523-549

Prabhakar M (2004). Structure, delimitation, nomenclature and classification of stomata. Acta Bot Sin Ed 46:242-252

Rapini A, C van den Berg, S Liede-Schumann (2007). Diversification of Asclepiadoideae (Apocynaceae) in the new world. Ann Missouri Bot Gard 94:407-422

Rapini A, MW Chase, DJ Goyder, J Griffiths (2003). Asclepiadeae classification: Evaluating the phylogenetic relationships of New World Asclepiadoideae (Apocynaceae). Taxon 52:33-50

Rohlf FJ (2000). NTSYS-PC Numerical Taxonomy and Multivariate Analysis System. version 2.1: Owner manual. New York, USA

Sennblad B, B Bremer (1996). The familial and subfamilial relationships ofApocynaceae andAsclepiadaceae evaluated withrbcL data. Plant Syst Evol 202:153-175

Sennblad B, B Bremer (2002). Classification of Apocynaceae s.1. according to a new approach combining Linnaean and phylogenetic taxonomy. Syst Biol 51:389-409

Simões AO, T Livshultz, E Conti, ME Endress (2007). Phylogeny and systematics of the Rauvolfioideae (Apocynaceae) based on molecular and morphological evidence. Ann Missouri Bot Gard 94:268-297

Stearn W (2005). Botanical latin. David \& Charles Publishers, London, UK

Tackholm V (1974). Supplementary Notes to Student's Flora of Egypt. Cairo University, Cairo, Egypt

Takhtajan AL (1980). Outline of the classification of flowering plants (Magnoliophyta). Bot Rev 46:225-359 